Research Paper

\title{
The Efficacy of Bevacizumab in Different Line Chemotherapy for Chinese Patients with Metastatic Colorectal Cancer
}

\author{
Chenxi Yin 1,2,3*, Gang Ma1,2,3* Yuming Rong1,2,4, Pengfei Kong1,2,4, Qiong Yang1,2,4, Chang Jiang 1,2,4, Fangxin \\ Liao $^{1,2,4}$, Bei Zhang ${ }^{1,2,4}$, Wenzhuo He ${ }^{1,2,4 \bowtie}$, Liangping Xia ${ }^{1,2,4 \bowtie}$ \\ 1. State Key Laboratory of Oncology in South China, Sun Yat-sen University Cancer Center, Guangzhou, Guangdong, 510060, R.P. China; \\ 2. Collaborative Innovation Center for Cancer Medicine, Guangzhou, Guangdong, 510060, R.P. China; \\ 3. ICU, Sun Yat-sen University Cancer Center, Guangzhou, Guangdong, 510060, R.P. China; \\ 4. VIP Region, Sun Yat-sen University Cancer Center, Guangzhou, Guangdong, 510060, R.P. China. \\ * contributed equally to this paper. \\ $\square$ Corresponding authors: Liangping Xia, Email: xialiangping@163.com Fax: +8620-87343392 Tel: +8620-87343107 Mobile: +86-13926410608 Co-corresponding \\ author: Wenzhuo He.
}

( ) Ivyspring International Publisher. Reproduction is permitted for personal, noncommercial use, provided that the article is in whole, unmodified, and properly cited. See http://ivyspring.com/terms for terms and conditions.

Received: 2016.04.11; Accepted: 2016.07.21; Published: 2016.09.13

\begin{abstract}
Objective: To evaluate the effect of bevacizumab in different lines for Chinese patients with metastatic colorectal cancer ( $\mathrm{mCRC}$ ).

Methods: Patients of $m C R C$ treated with bevacizumab or not at Sun Yat-sen University Cancer Center from 2007 to 2013 were recruited as study and control group. Endpoints were overall survival (OS), progression free survival (PFS), objective response rate (ORR) and disease control rate (DCR). Corresponding survival rates of first- and second-line in study and control group were compared.

Results: 1 . Median OS of study and control group were 44.8 (95\% Cl: $37.1 \sim 52.4)$ months, 36.1 $(95 \% \mathrm{Cl}$ : 32.8 39.5) months respectively, which were significantly different $(\mathrm{P}=0.004)$. 2 . In the first line treatment, median OS of study and control group were $49.9(95 \% \mathrm{Cl}: 40.1 \sim 59.8)$ months and 36.1 (95\% Cl: 32.7 39.4) months ( $P=0.002)$, respectively. And median PFS were 10.1( $95 \% \mathrm{Cl}$ : 8.7 11.5) months and 6.2 (95\% Cl: 5.5 6.8) months ( $\mathrm{P}<0.001)$, respectively. 3 . In the second line treatment, median OS of study and control group were 34.8 (95\% Cl: 26.3 43.3) months and 24.6 (95\% Cl: 22.2 27.0) months ( $\mathrm{P}=0.022)$, respectively. And the mPFS were $6.3(95 \% \mathrm{Cl}: 4.7 \sim 7.8)$ months and $3.1(95 \% \mathrm{Cl}$ : $2.5 \sim 3.6)$ months $(\mathrm{P}<0.001)$, respectively. 4. Median OS of first- and second-line treatment of the study groups were $49.9(95 \% \mathrm{Cl}: 40.1 \sim 59.8)$ months and 34.8 ( $95 \% \mathrm{Cl}$ : 26.3 43.3) months $(P=0.189)$, respectively.

Conclusion: The combination of bevacizumab and chemotherapy had a promising efficacy in Chinese mCRC patients. However, their OS were statistically insignificant between first- and second-line of bevacizumab groups.
\end{abstract}

Key words: bevacizumab; chemotherapy line setting; mCRC; efficacy.

\section{Introduction}

Bevacizumab, the classical anti-angiogenesis drug, had been expected to both prune the immature vessels and normalize tumor vessels by decreasing interstitial fluid pressure and increasing the delivery of drugs and oxygen[1]. It had been approved by the US Food and Drug Administration (FDA) to be used in various cancers such as metastatic colorectal cancer $(\mathrm{mCRC})^{[2-7]}$, advanced non-small cell lung cancer 
(NSCLC) $^{[8,9]}$, advanced renal cell carcinoma ${ }^{[10,11],}$ metastatic breast cancer ${ }^{[12,13]}$ and so on.

In metastatic colorectal cancer (mCRC), bevacizumab was widely used in the first- $[2,6]$, second- [7], later-line treatment ${ }^{[14]}$ and even progression beyond its failure[15]. However, the administration of bevacizumab had always been challenged. Though both of PFS and OS were improved significantly in AVF2017, ARTIST, and E3200 trials $[2,3,7]$, the original short OS of IFL regimen in the former two trials weakened their significances. Moreover, only PFS rather than OS was significantly improved in the NO16966 trial[16], which was also the main reason of FDA withdrawing the license of bevacizumab when combined with pacelitaxel in the first line setting of metastatic breast cancer ${ }^{[17-20]}$.

Bevacizumab is available in the treatment of first-, second-, later-line and even beyond its initial disease progression for $\mathrm{mCRC}$, and the efficacy might increasing with treatment course. However, not all patients had the opportunity to receive bevacizumab treatment. In the developing countries and the countries with incomplete health insurance, mCRC patients could only receive the expensive drug in just one-line of the whole treatment. Then, the current question is that which line therapy is more beneficial with the combination of bevacizumab? Current researches of it had not been found yet. A previous study of us had analyzed a small sample of Chinese mCRC, the result showed that the OS of bevacizumab combined group is much more longer than chemotherapy alone group, while it had no statistically difference between the first- and second-line of bevaciumab combined treatment ${ }^{[21]}$. The current study expanded the sample size and prolonged the follow-up time, and the aim of it is not only to confirm our previous result but also offer some valuable data to the mCRC patients with limited opportunity to receive bevacizumab treatment.

\section{Materials and methods}

\section{Study population}

Patients who met the following criterions were selected as study group: 1) Diagnosed as mCRC based on pathological specimens of the primary tumor, at the same time with clinical and/or pathological evidences of distant metastasis lesion, at the Sun Yat-sen University Cancer Center from 2007 to 2013 and finished the entire course of first- and second-line chemotherapy in this center 2) Eastern Cooperative Oncology Group (ECOG) performance status $\leq 2 ; 3$ ) Bevacizumab was added to the palliative regimens including oxaliplatin-based, irinotecan-based regimens and so on; 4) More than 4 cycles of bevacizumab was required, since 4 cycles was considered the minimum course for the patients got benefit from bevacizummab in our previous study[22]. Meanwhile, the consecutive mCRC patients in the control group met the same criterions with the study group except for bevacizumab administration. The exclusion criterions of both groups included 1) No pathological diagnosis; 2) Unclear medical histories; 3) Bevacizumab beyond its initial disease progression [15].

\section{Evaluation of efficacy}

The short-term effect was evaluated by the independent group based-on criterion of RESIST 1.1 criteria (Response Evaluation Criteria in Solid Tumors): overall response rate (ORR), disease control rate (DCR); the long-term efficacy was evaluated by comparing overall survival (OS) and progression free survival (PFS).

\section{Assessment}

Patients' OS, PFS, ORR and DCR were the primary statistical endpoints of the study. OS was calculated from diagnosis to death or the date of last follow-up (December 25 $\left.5^{\text {th }}, 2015\right)$, and PFS was deemed to be the period from the initial treatment of bevacizumab to the progression date by imaging examination according to the RECIST 1.1 criteria.

\section{Statistical analyses}

Survival curves were estimated using the Kaplan-Meier method. All the statistical analysis was conducted by SPSS 18.0 software package and the P value less than 0.05 was considered with statistically significant.

\section{Follow up}

The last follow-up was conducted on December $25^{\text {th }}, 2015$ through telephone interview or medical records review. All the patients in the study and the control group were followed up closely by then.

\section{Human Rights}

All the patients' information in our study has got IRB Approval of our hospital.

\section{Results}

\section{Patient characteristics and therapeutic regimens}

There were 82 patients entered the study group, all of them received bevacizumab $(5 \mathrm{mg} / \mathrm{kg}$, every two weeks) combined with chemotherapy in first- or second-line treatment. In the first-line of study group, there were 29 and 31 patients treated with oxaliplatin-based (FOLFOX and XELOX) and 
irinotecan-based (FOLFIRI, IFL, CPT-11 plus xeloda) chemotherapy, respectively; the median cycles of bevacizumab were 9.62 (ranged from $4 \sim 25$ ). In the second-line treamtment, 12 and 10 patients were treated with oxaliplatin-based and irinotecan-based chemotherapy, respectively; the median cycles of bevacizumab were 6.86 (ranged from 4 15). While in the first- and second-line of control group, 446 patients were treated with chemotherapy alone. Patients were treated with oxaliplatin-based, irinotecan-based and other chemotherapy as well, which is shown in Table 3. All the patients' characteristics and potential prognostic factors including sex, age, tumor location, pathological type and receiving third-line treatment or not were balanceable between the study group and the control group (Table 1). In the study group, $98.8 \%(81 / 82)$ and $42.7 \%(35 / 82)$ patients exhibited progressed and died, and $100 \%(446 / 446)$ patients and $64.6 \%$ $(288 / 446)$ patients exhibited progressed and died in the control group (Table 2).

Table 1. Patient baseline characteristics in the study and the control group.

\begin{tabular}{|c|c|c|c|c|c|}
\hline \multirow{2}{*}{$\begin{array}{l}\text { Patient } \\
\text { characteristics }\end{array}$} & \multirow[t]{2}{*}{ Control group } & \multirow[t]{2}{*}{ Study group } & \multicolumn{3}{|c|}{$\mathrm{P}^{*}$} \\
\hline & & & 1 & 2 & 3 \\
\hline Number & 446 & 82 & & & \\
\hline \multicolumn{6}{|l|}{ Gender } \\
\hline Male & $297(66.6 \%)$ & $53(64.6 \%)$ & 0.730 & 0.325 & 0.213 \\
\hline Female & $149(33.4 \%)$ & $29(35.4 \%)$ & & & \\
\hline \multicolumn{6}{|l|}{ Age (years) } \\
\hline Median (range) & $54(16 \sim 84)$ & $53(20 \sim 78)$ & 0.181 & 0.154 & 0.820 \\
\hline$\leq 65$ & $352(78.9 \%)$ & $70(85.4 \%)$ & & & \\
\hline$>65$ & $94(21.1 \%)$ & $12(14.6 \%)$ & & & \\
\hline \multicolumn{6}{|l|}{ Primary tumor } \\
\hline Colon & $295(66.1 \%)$ & $55(67.1 \%)$ & 0.870 & 0.583 & 0.222 \\
\hline Rectum & $151(33.9 \%)$ & $27(32.9 \%)$ & & & \\
\hline \multicolumn{6}{|l|}{ Pathological type } \\
\hline Adenocarcinoma & $413(92.6 \%)$ & $77(93.9 \%)$ & 0.675 & 0.907 & 0.628 \\
\hline Others & $33(7.4 \%)$ & $5(6.1 \%)$ & & & \\
\hline $\begin{array}{l}\text { Accepted three-line } \\
\text { treatment }\end{array}$ & & & 0.676 & 0.478 & 0.791 \\
\hline Yes & $106(23.8 \%)$ & $21(25.6 \%)$ & & & \\
\hline No & $340(76.2 \%)$ & $61(74.4 \%)$ & & & \\
\hline
\end{tabular}

Study group: the group of patients receiving bevacizumab.

Control group: the group of patients not receiving bevacizumab.

$\mathrm{P}^{*} 1$ : the balance test $\mathrm{P}$ value of total patients.

$P * 2$ : the balance test $P$ value of the first-line regimen.

$P * 3$ : the balance test $P$ value of the second-line regimen.

\section{Efficacy of bevacizumab in different lines}

In the entire group, the median OS of the study and the control group were 44.8 (95\% CI: 37.1 52.4) months, 36.1 (95\% CI: 32.8 39.5) months ( $\mathrm{P}=0.004)$, respectively (Fig.1).

The median OS of study group and control group in the first-line were 49.9(95\% CI: 40.1 59.8) months and $36.1 \quad(95 \%$ CI: 32.7 39.4) months
$(\mathrm{P}=0.002)$; in the second-line were 34.8 (95\% CI: 26.3 43.3) months and 24.6 (95\% CI: 22.2 27.0) months ( $\mathrm{P}=0.022)$, respectively (Fig.2, Fig.3).

The median PFS of the study group and control group in the first-line were $10.1(95 \%$ CI: 8.7 11.5) months and 6.2 (95\% CI: 5.5 6.8) months ( $\mathrm{P}<0.001)$; in the second-line were 6.3 (95\% CI: 4.7 7.8) months months and 3.1 (95\% CI: 2.5 3.6) months ( $\mathrm{P}<0.001)$, respectively (Fig.4, Fig.5).

In the study group, the meidan OS of the firstand second-line treatment were $49.9(95 \%$ CI: 40.1 59.8) and 34.8 (95\% CI: 26.3 43.3) months $(\mathrm{P}=0.189)$, respectively (Fig.6). The ORR were $30 \%$ (18/60), 22.7\% (5/22); DCR were 63.3\% (38/60), 59.1\% $(13 / 22)(\mathrm{P}=0.359)$, respectively (Table 2$)$.

Table 2. The efficacy of bevacizumab in the study group.

\begin{tabular}{lll}
\hline $\begin{array}{l}\text { Patient } \\
\text { characteristics }\end{array}$ & Bevacizumab group \\
\cline { 2 - 3 } & First-line & Second-line \\
\hline Number & 60 & 22 \\
ORR (\%) & $30 \%(18 / 60)$ & $22.7 \%(5 / 22)$ \\
DCR (\%) & $63.3 \%(38 / 60)$ & $59.1 \%(13 / 22)$ \\
PFS (months) & $10.1(95 \%$ CI: $8.7 \sim 11.5)$ & $6.3(95 \%$ CI: $4.7 \sim 7.8)$ \\
OS (months) & $49.9(95 \%$ CI: $40.1 \sim 59.8)$ & $34.8(95 \%$ CI: $26.3 \sim 43.3)$ \\
\hline
\end{tabular}

Table 3. The first and second-line chemotherapy regimen of control and study group

\begin{tabular}{lllll}
\hline Patient characteristics & \multicolumn{2}{l}{ First-line } & \multicolumn{2}{l}{ Second-line } \\
\cline { 2 - 5 } & Control & Study & Control & Study \\
\hline Number & 446 & 60 & 163 & 22 \\
Oxaliplatin based & 357 & 29 & 41 & 12 \\
Irinotecan based & 75 & 31 & 113 & 10 \\
Other chemotherapy & 14 & 0 & 9 & 0 \\
\hline Study group: the group of patients receiving bevacizumab; oxaliplatin-based: \\
FOLFOX, XELOX; irinotecan-based: FOLFIRI, IFL, CPT-11+xeloda. \\
Control group: the group of patients not receiving bevacizumab; oxaliplatin-based: \\
FOLFOX, XELOX, oxaliplatin+S-1; irinotecan-based: FOLFIRI, CPT-11+S-1
\end{tabular}

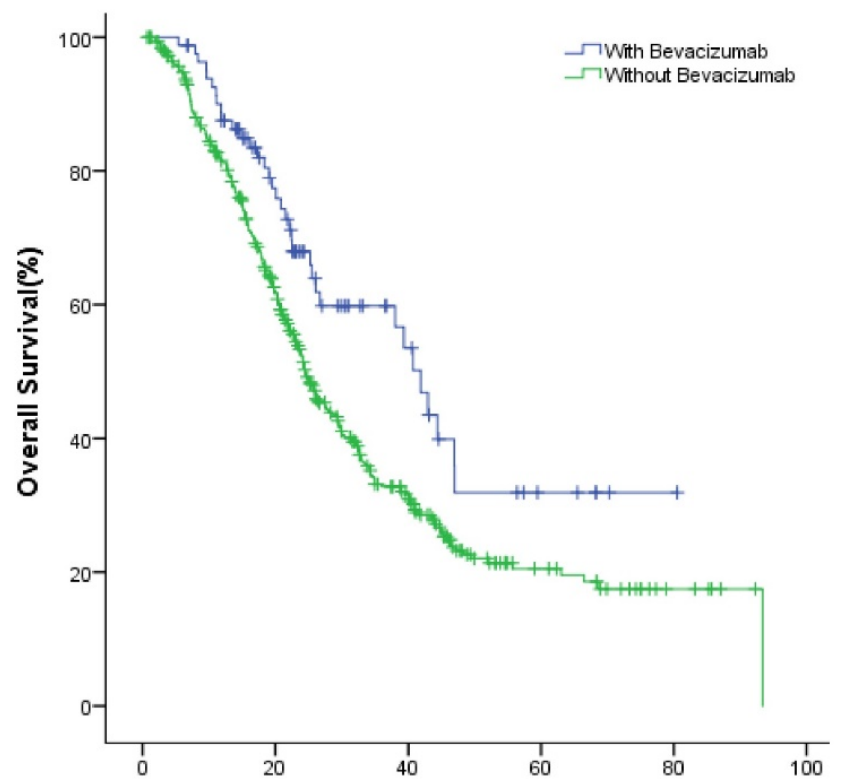

Figure 1: The OS of patients with or without bevacizumab. 


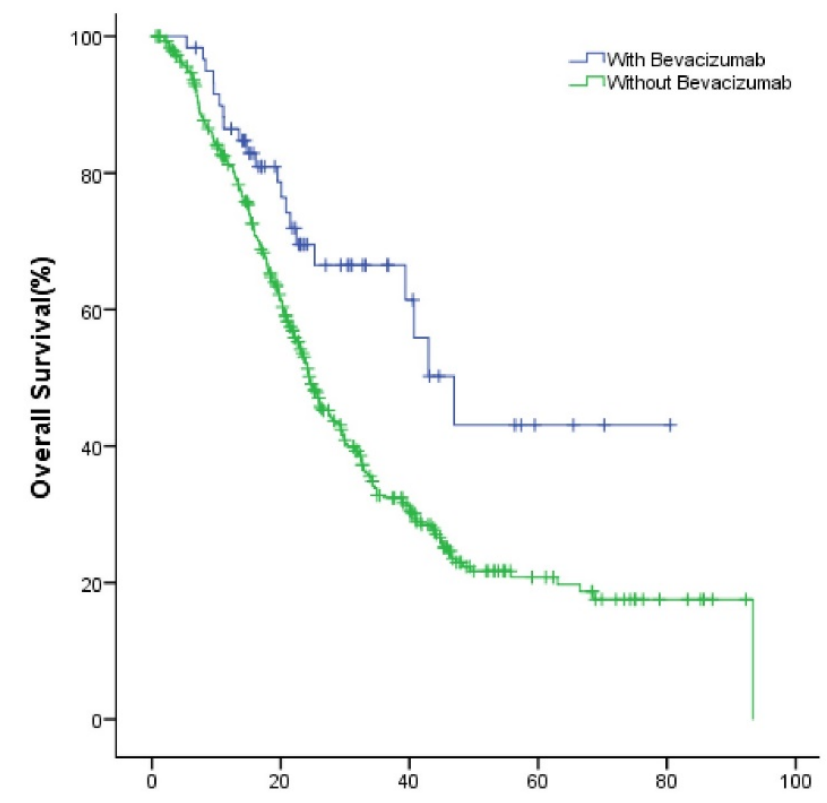

Figure 2: The OS of patients with and without bevacizumab in the first-line treatment.

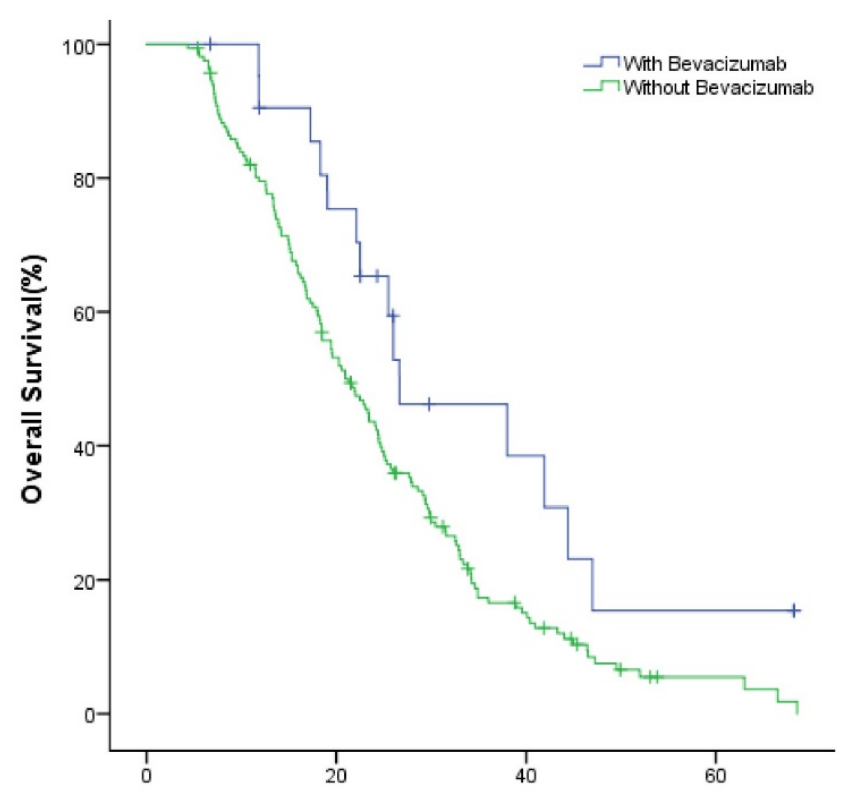

Figure 3: The OS of patients with and without bevacizumab in the second-line treatment.

\section{Discussion}

The similar effect of bevacizumab in Caucasian mCRC patients and Chinese mCRC patients was indicated in clinical trials of AVF2017[2] and ARTIST ${ }^{[3]}$. However, In Chinese mCRC patients, the experiences of bevacizumab need accumulating. The early one-armed study of bevaczimab in Chinese mCRC patients found higher mortality and progressive rate (56.3\% and $40.6 \%$, respectively), which may relate to small patient sample. Later, both a recent study ${ }^{[23]}$ and our data[22, 24, 25] showed that OS was significantly

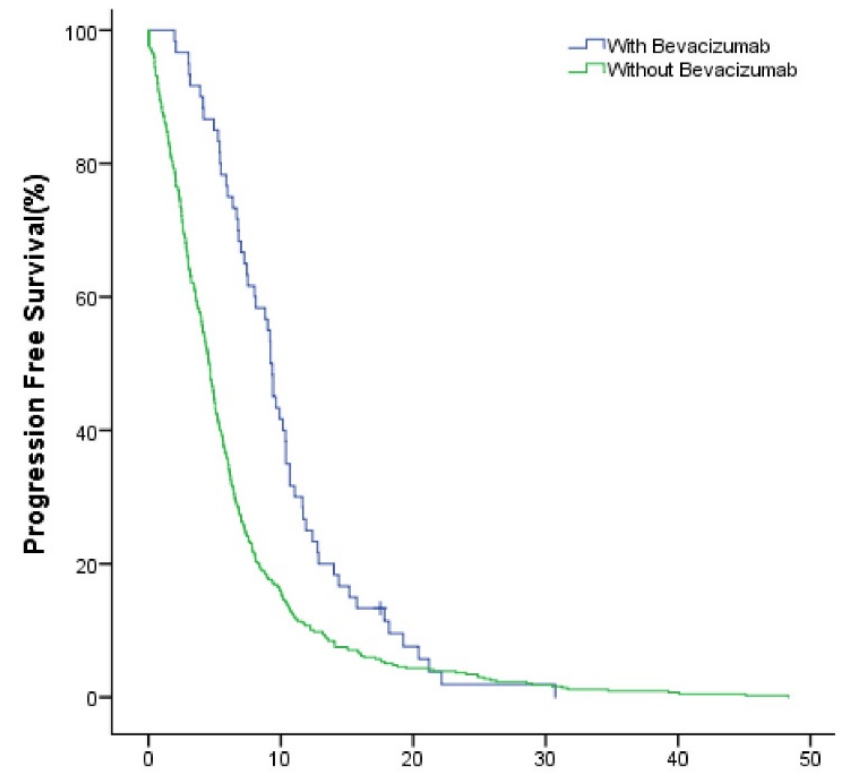

Figure 4: The PFS of patients with and without bevacizumab in the first-line treatment.

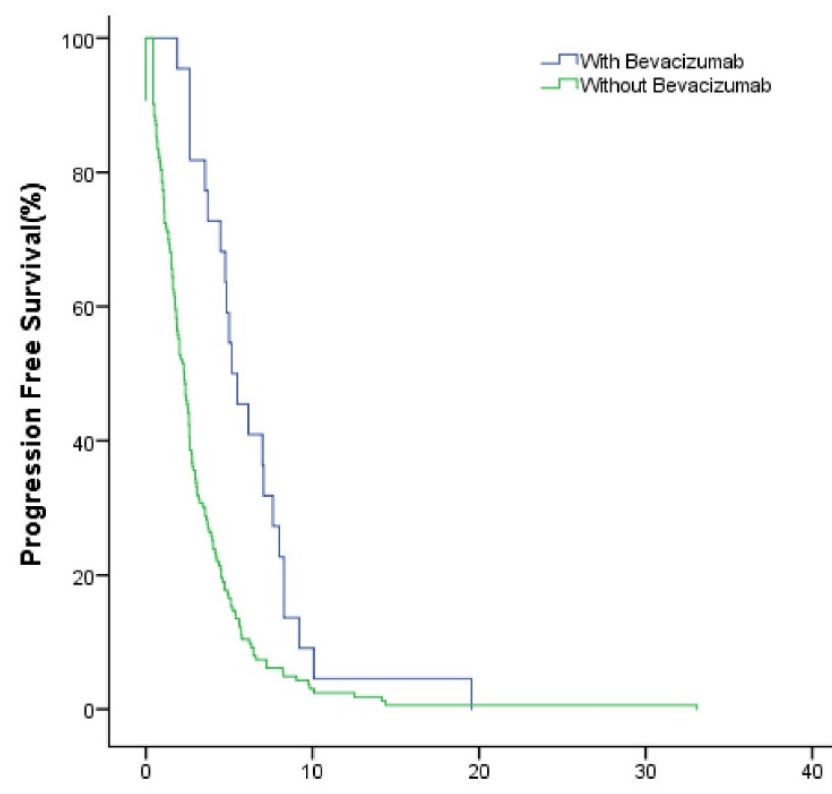

Figure 5: The PFS of patients with and without bevacizumab in the second-line treatment.

prolonged after the administration of bevacizumab in mCRC, even in the later line ${ }^{[14]}$. In addition, our studies also found that only patients received more than 4 cycles of bevacizumab can get survival benefit[22] and bevacizumab benefit only refine to patients with initial LDH increase before treatment.

To our best knowledge, the current study was the first study to compare the effect of bevacizumab among different lines treatment when combined with chemotherapy in mCRC. It was shown that not only ORR and DCR but also OS and PFS were promising 
after the addition of bevacizumab in each line treatment. In the entire group, the OS was prolonged indeed with the addition of bevacizumab when compared with patients without bevacizumab. Furthermore, both of OS and PFS were significant improved in bevacizumab group when compared with corresponding line chemotherapy alone groups. However, the OS were statistically insignificant among different line subgroups of bevacizumab group. All the patients in our study only received one line bevacizumab treatment since the patients received bevacizumab beyond its initial progression were excluded. Meanwhile, the current data, accordant with other studies, demostrated that the combination of bevacizumab and standard chemotherapy really brought survival benefit to mCRC patients no matter it was added in the first- or the second-line treatment. The exact cause is unknown, but a similar finding was seen in non-small cell lung cancer(NSCLC) patients who were EGFR sensitive mutations and treated with tyrosine kinase inhibitors(TKIs) ${ }^{[26]}$. Both clinical trials of IPASS and NEJSG002 [27, 28] were to investigate the sequence of gefitinib, the first generation of TKIs and chemotherapy in the first line and second line in patients with EGFR sensitive mutation. The OS were identical no matter the first- and second- line strategy was chemotherapy behind TKIs or the opposite, despite that the PFS of TKIs group was longer than chemotherapy in the first line. Therefore, the principle of TKIs administration was that it could be used in no matter which line. The reason of this phenomenon was unknown either in NSCLC patients as well.

Besides, the limits of this study should be taken into account before drawing a conclusion. Firstly, the retrospective data and small patients sample may cause bias. In addition, although this strategy were proven to be effective and be part of clinical practice already, the patients treated with bevacizumab beyond its initial progression were not covered in this study. The aim of excluding those patients was to guarantee the drug was received once in the patients of the study group during the whole course of treatment. As a consequence, it was possible to be compared with the patients in chemotherapy alone group. Moreover, only patients received more than four cycles of bevacizumab were recruited, since our previous study found the survival benefit refine to those group of mCRC patients.

In conclusion, once the patients received bevacizumab during the whole course of treatment, it really brought survival benefit to $\mathrm{mCRC}$ patients in no matter which line therapy. Nevertheless, the prospective and/or larger patients sample studies are urgently needed.

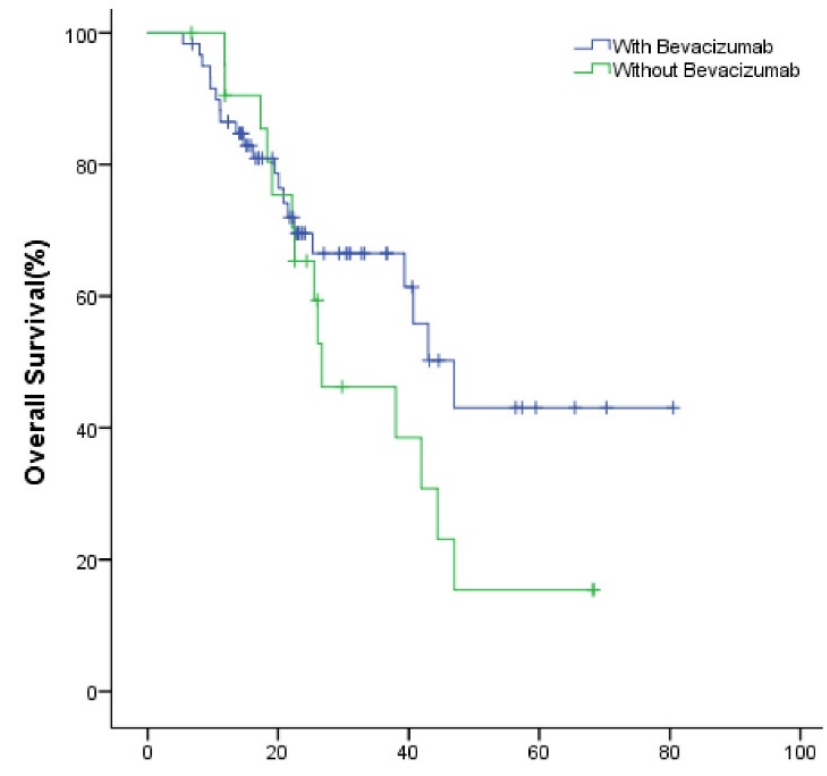

Figure 6: The OS of patients with bevacizumab in the first- and second-line treatment.

\section{Acknowledges}

This study was supported by grants from the Natural Science Foundation of Guangdong, China (2015A030313010), Science and Technology Program of Guangzhou, China (1563000305) and National Natural Science Foundation of China (81272641and 81572409).

\section{Competing Interests}

The authors have declared that no competing interest exists.

\section{References}

[1] Jain R K. Normalizing tumor vasculature with anti-angiogenic therapy: a new paradigm for combination therapy[J]. Nat Med. 2001, 7(9): 987-989.

[2] Hurwitz H, Fehrenbacher L, Novotny W, et al. Bevacizumab plus irinotecan, fluorouracil, and leucovorin for metastatic colorectal cancer[J]. N Engl J Med. 2004, 350(23): 2335-2342.

[3] Guan Z Z, Xu J M, Luo R C, et al. Efficacy and safety of bevacizumab plus chemotherapy in Chinese patients with metastatic colorectal cancer: a randomized phase III ARTIST trial[J]. Chin J Cancer. 2011, 30(10): 682-689.

[4] Fuchs C S, Marshall J, Mitchell E, et al. Randomized, controlled trial of irinotecan plus infusional, bolus, or oral fluoropyrimidines in first-line treatment of metastatic colorectal cancer: results from the BICC-C Study[J]. J Clin Oncol. 2007, 25(30): 4779-4786.

[5] Hochster H S, Hart L L, Ramanathan R K, et al. Safety and efficacy of oxaliplatin and fluoropyrimidine regimens with or without bevacizumab as first-line treatment of metastatic colorectal cancer: results of the TREE Study[J]. J Clin Oncol. 2008, 26(21): 3523-3529.

[6] Saltz L B, Clarke S, Diaz-Rubio E, et al. Bevacizumab in combination with oxaliplatin-based chemotherapy as first-line therapy in metastatic colorectal cancer: a randomized phase III study[J]. J Clin Oncol. 2008, 26(12): 2013-2019.

[7] Giantonio B J, Catalano P J, Meropol N J, et al. Bevacizumab in combination with oxaliplatin, fluorouracil, and leucovorin (FOLFOX4) for previously treated metastatic colorectal cancer: results from the Eastern Cooperative Oncology Group Study E3200[J]. J Clin Oncol. 2007, 25(12): 1539-1544.

[8] Sandler A, Gray R, Perry M C, et al. Paclitaxel-carboplatin alone or with bevacizumab for non-small-cell lung cancer[J]. N Engl J Med. 2006, 355(24): 2542-2550.

[9] Reck M, von Pawel J, Zatloukal P, et al. Phase III trial of cisplatin plus gemcitabine with either placebo or bevacizumab as first-line therapy for nonsquamous non-small-cell lung cancer: AVAil[J]. J Clin Oncol. 2009, 27(8): 1227-1234. 
[10] Escudier B, Pluzanska A, Koralewski P, et al. Bevacizumab plus interferon alfa-2a for treatment of metastatic renal cell carcinoma: a randomised, double-blind phase III trial[J]. Lancet. 2007, 370(9605): 2103-2111.

[11] Rini B I, Halabi S, Rosenberg J E, et al. Bevacizumab plus interferon alfa compared with interferon alfa monotherapy in patients with metastatic renal cell carcinoma: CALGB 90206[J]. J Clin Oncol. 2008, 26(33): 5422-5428.

[12] Gray R, Bhattacharya S, Bowden C, et al. Independent review of E2100: a phase III trial of bevacizumab plus paclitaxel versus paclitaxel in women with metastatic breast cancer[J]. J Clin Oncol. 2009, 27(30): 4966-4972.

[13] Miles D W, Chan A, Dirix L Y, et al. Phase III study of bevacizumab plus docetaxel compared with placebo plus docetaxel for the first-line treatment of human epidermal growth factor receptor 2-negative metastatic breast cancer[J]. J Clin Oncol. 2010, 28(20): 3239-3247.

[14] Yang Q, Yin C, Liao F, et al. Bevacizumab plus chemotherapy as third- or later-line therapy in patients with heavily treated metastatic colorectal cancer[J]. Onco Targets Ther. 2015, 8: 2407-2413.

[15] Bennouna J, Sastre J, Arnold D, et al. Continuation of bevacizumab after first progression in metastatic colorectal cancer (ML18147): a randomised phase 3 trial[J]. Lancet Oncol. 2013, 14(1): 29-37.

[16] Tyagi P, Grothey A. Commentary on a phase III trial of bevacizumab plus XELOX or FOLFOX4 for first-line treatment of metastatic colorectal cancer: the NO16966 trial[J]. Clin Colorectal Cancer. 2006, 6(4): 261-264.

[17] Rugo H S. Inhibiting angiogenesis in breast cancer: the beginning of the end or the end of the beginning? [J]. J Clin Oncol. 2012, 30(9): 898-901.

[18] Miles D W, Chan A, Dirix L Y, et al. Phase III study of bevacizumab plus docetaxel compared with placebo plus docetaxel for the first-line treatment of human epidermal growth factor receptor 2-negative metastatic breast cancer[J]. J Clin Oncol. 2010, 28(20): 3239-3247.

[19] Robert N J, Dieras V, Glaspy J, et al. RIBBON-1: randomized, double-blind, placebo-controlled, phase III trial of chemotherapy with or without bevacizumab for first-line treatment of human epidermal growth factor receptor 2-negative, locally recurrent or metastatic breast cancer[J]. J Clin Oncol. 2011, 29(10): 1252-1260.

[20] Sasich L D, Sukkari S R. The US FDAs withdrawal of the breast cancer indication for Avastin (bevacizumab)[J]. Saudi Pharm J. 2012, 20(4): 381-385.

[21] Yin C, Jiang C, Liao F, et al. The efficacy of bevacizumab in Chinese patients with metastatic colorectal cancer and its effect in different line setting[J]. Chinese-German Journal of Clinical Oncology. 2014, 4(13): 169-173.

[22] Zhang B, He W, Zhou F. A potential administration-time dependent effect of Bevacizumab in improving overall survival and increasing metastasis in metastatic colorectal cancer[J]. Chemotherapy. 2013: doi: 10.4172/2167-7700.1000108, 108.

[23] Bai L, Wang F, Zhang D S, et al. A plasma cytokine and angiogenic factor (CAF) analysis for selection of bevacizumab therapy in patients with metastatic colorectal cancer[J]. Sci Rep. 2015, 5: 17717.

[24] Yin C, Jiang C, Liao F, et al. Initial LDH level can predict the survival benefit from bevacizumab in the first-line setting in Chinese patients with metastatic colorectal cancer[J]. Onco Targets Ther. 2014, 7: 1415-1422.

[25] Yin C, Jiang C, Liao F, et al. The efficacy of bevacizumab in Chinese patients with metastatic colorectal cancer and its effect in different line setting[J]. Chinese-German J Clin Oncol. 2014, 13(4): 169-173.

[26] Yoshimura N, Kudoh S, Mitsuoka S, et al. Phase II study of a combination regimen of gefitinib and pemetrexed as first-line treatment in patients with advanced non-small cell lung cancer harboring a sensitive EGFR mutation[J]. Lung Cancer. 2015, 90(1): 65-70.

[27] Mok T S, Wu Y L, Thongprasert S, et al. Gefitinib or carboplatin-paclitaxel in pulmonary adenocarcinoma[J]. N Engl J Med. 2009, 361(10): 947-957.

[28] Maemondo M, Inoue A, Kobayashi K, et al. Gefitinib or chemotherapy for non-small-cell lung cancer with mutated EGFR[J]. N Engl J Med. 2010, 362(25): 2380-2388. 